Pacific Journal of Mathematics

TERATIONS AND FIXPOINTS 


\section{ITERATIONS AND FIXPOINTS}

\section{BENNO FUCHSSTEINER}

Starting with a decreasing map $\varphi: X \rightarrow X$ on a partially ordered set $X$ we construct a map It $\varphi$ which intuitively can be understood as the iteration (countable or transfinite) of $\varphi$. The main properties which It $\varphi$ inherits from $\varphi$ are investigated. As application of the main result some fixpoint theorems are proved. Besides, our method yields constructive proofs for results which are usually demonstrated with the help of the axiom of choice.

I. The iteration theorem. We consider a partially ordered set $(X, \leqq)$ and a decreasing map $\varphi: X \rightarrow X$. For $Y \subset X$ we denote by $\min (Y)$ the set of minimal elements in $Y$. A totally ordered subset $Y \subset X$ is said to be a $\varphi$-chain if:

$$
\begin{gathered}
\text { (C1) } y \leqq \varphi(x) \text { for all } x, y \in Y \text { with } x \neq y \text { and } y \leqq x \\
(\text { C } 2)^{+} \varphi(Y \backslash \min (Y)) \subset Y .
\end{gathered}
$$

$(X, \leqq)$ is assumed to be $\varphi$-complete, which means that every nonempty $\varphi$-chain has an infimum. A $\varphi$-chain $Y$ is called a strong $\varphi$-chain if:

$$
(\mathrm{C} 2)^{*} \varphi(\inf (\bar{Y})) \in Y \text { for all nonempty } \bar{Y} \subset Y \text { with } \inf (\bar{Y}) \neq \inf (Y) \text {. }
$$

Condition (C2)* obviously implies $(\mathrm{C} 2)^{+}$. Elementary examples for strong $\varphi$-chains are $\{x\}$ and $\{x, \varphi(x)\}$. A strong $\varphi$-chain $Y$ is said to be a complete $\varphi$-chain if $\varphi(\inf (Y)) \in Y$.

Remark 1. (i) A totally ordered subset $Y \subset X$ is a complete $\varphi$-chain iff (C1) and

$$
\text { (C2) } \varphi(\inf (\bar{Y})) \in Y \text { for all nonempty } \bar{Y} \subset Y \text {. }
$$

(ii) The intersection of a family of strong (complete) $\varphi$-chains is a strong (complete) $\varphi$-chain.

LEMMA 1. Every strong $\varphi$-chain $Y$ is contained in a complete $\varphi$-chain. The complete $\varphi$-chains containing $Y$ have a minimum $I_{Y}$ with respect to $\subset$. 
Proof. Let $M$ be the set of strong $\varphi$-chains containing $Y$. We define $\bar{\varphi}: M \rightarrow M$ by $\bar{\varphi}(\tilde{Y})=\tilde{Y} \cup\{\varphi(\inf (\tilde{Y}))\}$. According to Zermelo's fixpoint-lemma [4, Theorem I.2.5] $\bar{\varphi}$ has a fixpoint, which is by definition a complete $\varphi$-chain. $I_{Y}$ is obviously given by $\cap\{\tilde{Y} \in M \mid \bar{\varphi}(\tilde{Y})=\tilde{Y}\}{ }^{*}$

A $\varphi$-invariant subset $Y \subset X$ is called a $\varphi$-subset if the infimum of every strong $\varphi$-chain in $Y$ is an element of $Y$. A map $F: X \rightarrow X$ is called $\varphi$-absorbing if:

(A 1) $F \circ \varphi=F$

(A 2) if $F$ is constant on a nonempty strong $\varphi$-chain $Y$ then $F$ is constant on $Y \cup\{\inf (Y)\}$.

Iteration TheOREM. There is a decreasing map It $\varphi: X \rightarrow X$ such that:

(i) It $\varphi$ maps onto the $\varphi$-fixpoints of $X$

(ii) It $\varphi \circ$ It $\varphi=$ It $\varphi$

(iii) every $\varphi$-subset is It $\varphi$-invariant

(iv) for every $\varphi$-absorbing map $F$ we have $F \circ$ It $\varphi=F$

(v) for every $\varphi$-absorbing map $F$ with $F(x) \leqq x \Rightarrow F(x) \leqq \varphi(x)$ we have $F(x) \leqq x \Rightarrow F(x) \leqq$ It $\varphi(x)$.

(vi) It $\varphi$ is $\varphi$-absorbing.

It $\varphi$ is uniquely determined by $\varphi$.

Proof. We define It $\varphi(x) \doteqdot \inf \left(I_{x}\right)$ where $I_{x} \supset\{x\}$ is the minimal complete $\varphi$-chain given by Lemma 1 . We claim that It $\varphi$ is constant on $I_{x}$.

Proof of the claim. Let $y_{0} \in I_{x}$. Then $\left\{y \in I_{x} \mid y \leqq y_{0}\right\}$ and $\left\{y \in I_{x} \mid y \geqq y_{0}\right\} \cup I_{y_{0}}$ are complete $\varphi$-chains $\supset\left\{y_{0}\right\}$ and $\supset\{x\}$ respectively. Hence from minimality of $I_{x}$ and $I_{y_{0}}$ it follows $I_{x}=$ $\left\{y \in I_{x} \mid y \geqq y_{0}\right\} \cup I_{y 0}$.

This implies It $\varphi\left(y_{0}\right)=\inf \left(I_{y_{0}}\right)=\inf \left(I_{x}\right)=$ It $\varphi(x)$.

(i) and (ii): It $\varphi(x)=\inf \left(I_{x}\right)$ is a $\varphi$-fixpoint because of $\inf \left(I_{x}\right) \geqq$ $\varphi\left(\inf \left(I_{x}\right)\right) \in I_{x}$. Now, let $x=\varphi(x)$ then $I_{x}=\{x\}$ (minimality). This implies It $\varphi(x)=\inf \left(I_{x}\right)=x$. Hence It $\varphi$ maps onto the fixpoints and is idempotent.

(iii): Let $x \in Y$ where $Y$ is a $\varphi$-subset. Then $\bar{I}_{x} \doteqdot$ $\left\{y \in I_{x} \mid\left\{\xi \in I_{x} \mid y \leqq \xi\right\} \subset Y\right\}$ is a complete $\varphi$-chain. We have $x \in \bar{I}_{x}$ because $\xi \leqq x \forall \xi \in I_{x}$. Hence $\bar{I}_{x}=I_{x} \quad$ (minimality) and $\operatorname{It} \varphi(x)=$ $\inf \left(I_{x}\right) \in \bar{I}_{x} \subset Y$.

* The fixpoint-lemma is applied with respect to the following order in $M$ : $Y_{1}<Y_{2} \Leftrightarrow Y_{1}$ is ideal of $Y_{2}$, i.e. $Y_{1} \subset Y_{2}$ and $y \leqq x \forall y \in Y_{2} \backslash Y_{1}, x \in Y_{1}$. 
(iv): Consider $\bar{I}_{x}=\left\{y \in I_{x} \mid F(x)=F(\xi) \forall \xi \in I_{x}\right.$ with $\left.\xi \geqq y\right\}$ where $F$ is $\varphi$-absorbing. $\bar{I}_{x}$ is a complete $\varphi$-chain $\supset\{x\}$. Hence $I_{x}=\bar{I}_{x}$ and It $\varphi(x) \in \bar{I}_{x}$. This implies $F \circ$ It $\varphi(x)=F(x)$.

(v): Let $F$ be as in (v) and $F(x) \leqq x$. Then $\bar{I}_{x}=\left\{y \in I_{x} \mid y \geqq\right.$ $F(y)=F(x)\}$ is a complete $\varphi$-chain $\supset\{x\}$. This gives $I_{x}=\bar{I}_{x}$ and $F(x)=F\left(\inf \left(I_{x}\right)\right) \leqq \inf \left(I_{x}\right)=$ It $\varphi(x)$.

(vi): (A 1) is a consequence of $\varphi(x) \in I_{x}$ and the fact that It $\varphi$ is constant on $I_{x}$. Now, let $x \in Y$ where $Y$ is a strong $\varphi$-chain on which It $\varphi$ is constant. For $\bar{x}=\inf (Y)$ we show that $I_{x} \cap I_{\bar{x}} \neq \varnothing$. This gives the desired result because for $y \in I_{x} \cap I_{\tilde{x}}$ we have It $\varphi(x)=\operatorname{It} \varphi(y)=$ It $\varphi(\bar{x})=$ It $\varphi(\inf Y)$. Assume therefore that $I_{x} \cap I_{\bar{x}}=\varnothing$. The set $Y \cup I_{\bar{x}}$ is a complete $\varphi$-chain $\supset\{x\}$. Hence $Y \cup I_{\bar{x}} \geqq I_{x}$ and $I_{x}$ must be a subset of $Y$. This gives $\bar{x} \rightrightarrows \inf \left(I_{x}\right)$ ( $\neq$ because of $\left.\bar{x} \notin I_{x}\right)$. Since $Y \supset I_{x}$ is totally ordered there is some $y \in Y$ with $y \lessgtr \inf \left(I_{x}\right)$. From this it follows It $\varphi(y) \leqq y \lessgtr \inf \left(I_{x}\right)=$ It $\varphi(x)$ which is in contradiction to the fact that It $\varphi$ is constant on $Y$.

Proof of uniqueness. Let $F$ be $\varphi$-absorbing and decreasing then according to (v) we have $F(x) \leqq$ It $\varphi(x)$ for all $x$. Thus It $\varphi$ is the maximum of all decreasing $\varphi$-absorbing maps. Hence It $\varphi$ is unique.

From now on It $\varphi$ is called the iteration of $\varphi$. The reason for choosing this name will become obvious in the next chapter. It should be mentioned that the proof of the iteration theorem does not make use of Zorn's lemma or the axiom of choice. The crucial tool was Zermelo's fixpoint-lemma (see also [15]) which can be proved constructively.

\section{Examples and applications.}

II.1. Monotony and a theorem of Tarski. First we show that It $\varphi$ inherits monotony from $\varphi$.

LeMMA 2. Let $\varphi$ be monotone.

(i) It $\varphi(x)$ is the maximum of the $\varphi$-fixpoints $\leqq x$.

(ii) It $\varphi$ is monotone.

Proof. (i) Let $x_{0}$ be a fixpoint $\leqq x$. We show $x_{0} \leqq$ It $\varphi(x)$. Obviously the constant map $F: X \rightarrow X$ given by $F(y)=x_{0}$ is $\varphi$-absorbing. $x_{0} \leqq y$ implies $\varphi\left(x_{0}\right)=x_{0} \leqq \varphi(y)$ because $\varphi$ is monotone. Hence $F$ fulfills (v) of the iteration theorem and we have $x_{0}=F(x) \leqq$ It $\varphi(x)$.

(ii) Let $y \leqq x$ then It $\varphi(y)$ is a $\varphi$-fixpoint $\leqq x$. By (i) we know It $\varphi(y) \leqq$ It $\varphi(x)$. Thus It $\varphi$ must be monotone.

ExAmple 1. Let $L$ be a complete lattice and $\mu: L \rightarrow L$ a 
monotone map. $\quad \mu$ is decreasing on $X \doteqdot\{x \in L \mid \mu(x) \leqq x\}$ which is not empty because $\sup (L) \in X$.

$X$ contains Fix $(\mu)$ (set of $\mu$-fixpoints).

$X$ is $\mu$-complete because the infimum of every chain in $X$ is again element of $X$. This yields that $\operatorname{Fix}(\mu) \neq \varnothing$ (see [9]) because $\operatorname{Fix}(\mu)$ is the image of $X$ under It $\mu_{\mid X}$.

Now, let $Y \subset \operatorname{Fix}(\mu)$ then by Lemma 2(i) It $\mu_{\mid X}(\inf (Y))$ is the maximum of all fixpoints $\leqq \inf (Y)$. Hence It $\mu_{\mid X}(\inf (Y))$ is the infimum of $Y$ in $\operatorname{Fix}(\mu)$. So we have proved:

THEOREM (Tarski [14]). Let $\mu: L \rightarrow L$ be monotone on the complete lattice $L$, then $\operatorname{Fix}(\mu)$ is a nonempty complete lattice.

II.2. Contracting and condensing maps. The next example is rather trivial. Nevertheless it shows why the name iteration was chosen for It $\varphi$. Let $(Z, d)$ be a complete and bounded metric space. We call $T: Z \rightarrow Z$ a generalized contraction if there is a $q<1$ such that $\delta(T(A)) \leqq q \delta(A)$ for all $T$-invariant $A \subset Z$, where $\delta()$ denotes the diameter. On $X=\{A \subset Z \mid A$ closed, nonempty, $T$-invariant $\}$ we define a $C$-decreasing map $\varphi_{T}$ by $\varphi_{T}(A)=$ closure $(T(A))$. Then $X$ is $\varphi_{T^{-}}$ complete because a $\varphi_{T}$-chain either contains only finitely many elements or is the basis of a Cauchy filter. It $\varphi_{T}(X)$ is a singleton because as $\varphi_{T}$-fixpoints it has diameter 0 . Furthermore It $\varphi_{T}(X)$ contains (Lemma 2(i)) the closure of the set of $T$-fixpoints. Hence the iteration theorem yields:

THEOREM (Banach and others). Every generalized contraction on $Z$ has a unique fixpoint.

Generalized contractions are for example the quasicontractions

$$
\begin{aligned}
& d(T(x), T(y)) \\
& \quad \leqq \\
& \quad \underset{\max }{\forall x, y \in Z}\{d(x, y), d(x, T(x)), d(y, T(y)), d(x, T(y)), d(y, T(x))\}
\end{aligned}
$$

recently considered [3] by Lj. B. Ćirić.

The contraction property in the last example was mainly used to assure that $X$ is $\varphi_{T}$-complete. But if $(Z, d)$ is compact then $X$ is $\varphi_{T}$-complete for any map $T$. Therefore the same argument leads to:

THEOREM (M. Edelstein [5]). Every condensing map T (i.e. a map with $\delta(T(A))<\delta(A)$ for every $T$-invariant $A \subset Z$, with $\delta(A)>0$ ) on $a$ compact metric space has a unique fixpoint. 
For the next example let us consider a continuous map $T: S \rightarrow S$, where $S$ is a nonempty closed bounded convex subset of a separated locally convex vector space. $T$ is said to be limit-compact if every closed $M \subset S$ with $\overline{c o} T(M) \supset M$ has to be compact. ( $\overline{c o}$ denotes the closed convex hull).

THEOREM (Sadovski [13]). Let T be limit-compact and assume that there is some nonempty $A \subset S$ with $\overline{c o} T(A) \supset A$. Then Thas a fixpoint.

Proof. On $X=\{Y \subset S \mid \overline{\mathrm{co}} Y=Y\}$ we consider the $C$-decreasing map $\varphi(Y) \doteqdot Y \cap \overline{c o} T(Y)$ and the constant map $F(Y) \doteqdot \overline{c o}(A) . \quad X$ is a complete lattice with respect to $C$ and therefore $\varphi$-complete. The $\varphi$-fixpoint $K \doteqdot$ It $\varphi(S)$ is compact because $T$ is limit-compact. (v) of the iteration theorem gives $\varnothing \neq \overline{\operatorname{co}}(A)=F(S) \subset$ It $\varphi(S)=K$. So $K$ is nonempty. Finally we get $T(K) \subset K$ from the fact that $\bar{X}=$ $\{Y \in X \mid T(Y) \subset Y\}$ is a $\varphi$-subset. Hence the Schauder-Tychonoff Theorem [4, Theorem, V.10.5] provides a fixpoint.

A special case of this theorem is that every continuous $\Delta$-condensing operator on a closed bounded convex set has a fixpoint ( $\Delta$ is a measure of noncompactness, see [12]).

II.3. Normal structure. Throughout this chapter $S$ will be a nonempty weakly compact set with normal structure in a Banach space.

Normal structure means that if $M$ is a convex subset of $S$ which contains more than one element then it holds that

$$
r(M) \doteqdot \inf _{z \in M} \sup _{x \in M}\|x-z\|<\delta(M) \doteqdot \sup _{z, x \in M}\|x-z\|
$$

We know that for every nonempty closed convex subset $M \subset S$ the Čebyčev-centre of $M$ with respect to $S \operatorname{Ceb}_{S}(M) \doteqdot$ $\left\{x \in S \mid \sup _{z \in M}\|x-z\| \leqq r(M)\right\}$ is again nonempty closed and convex (see [2], [8]). We shall write $\mathrm{Ceb}_{\bar{M}}(M)$ for $\operatorname{Ceb}_{S}(M) \cap \tilde{M}$ and $\operatorname{Ceb}(M)$ for $\operatorname{Ceb}_{M}(M)$. $\operatorname{Ceb}_{s}(M) \supset M \neq \varnothing$, or equivalently $\operatorname{Ceb}(M)=M \neq \varnothing$, happens if and only if $M$ is a singleton. We are interested in nonexpansive maps $T: S \rightarrow S$. Nonexpansive means that $\|T(x)-T(y)\| \leqq$ $\|x-y\|$ for all $x, y \in S$. It is well known ([6], [8]) that with additional conditions $\operatorname{Ceb}_{s}(M)$ is invariant under a nonexpansive map. We need this result in a slightly more general form.

LEMMA 3. Let $f: S \rightarrow S$ be nonexpansive and $M \subset S$ such that $\overline{\mathrm{co}} f(M) \supset M=\overline{\mathrm{co}}(M)$. Then $\mathrm{Ceb}_{s}(M)$ is $f$-invariant. 
Proof. Let $x \in M$ and $x_{0} \in \mathrm{Ceb}_{s}(M)$ be arbitrary. By assumption for $\epsilon>0$ there are $\lambda_{1}, \cdots, \lambda_{n} \geqq 0$ with $\Sigma \lambda_{t}=1$ and $y_{1}, \cdots, y_{n} \in M$ such that:

$$
\begin{aligned}
& \left\|f\left(x_{0}\right)-x\right\| \leqq\left\|f\left(x_{0}\right)-\Sigma \lambda_{i} f\left(y_{i}\right)\right\|+\epsilon \leqq \Sigma \lambda_{l}\left\|f\left(x_{0}\right)-f\left(y_{i}\right)\right\|+\epsilon \\
& \quad \leqq \Sigma \lambda_{i}\left\|x_{0}-y_{i}\right\|+\epsilon \quad \text { (because } f \text { is nonexpansive) } \\
& \quad \leqq r(M)+\epsilon .
\end{aligned}
$$

This implies $\left\|f\left(x_{0}\right)-x\right\| \leqq r(M) \forall x \in M$ and $f\left(x_{0}\right)$ must be in $\operatorname{Ceb}_{s}(M)$.

THEOREM (Kirk [8]). Every nonexpansive $T: S \rightarrow$ S has a fixpoint.

Proof. $X=\{Y \subset S \mid \varnothing \neq Y=\overline{\mathrm{co}}(Y), T(Y) \subset Y\}$ is $\varphi$-complete for every decreasing $\varphi$ because it consists of weakly compact sets. We define a decreasing $\varphi_{1}: X \rightarrow X$ by $\varphi_{1}(Y)=\overline{\text { co }} T(Y)$. The Čebyčev centre of It $\varphi_{1}(Y)$ is $T$-invariant because of Lemma 3 .

Thus $\varphi_{2}(Y) \doteqdot \operatorname{Ceb}\left(\right.$ It $\left.\varphi_{1}(Y)\right)$ is a decreasing map $X \rightarrow X$ and $K=$ It $\varphi_{2}(S)$ must be an element of $X$ with $\operatorname{Ceb}(K)=K$. Hence $K$ is a $T$-invariant singleton. That means $K$ consists of one $T$-fixpoint.

We close this section with a theorem of the Belluce-Kirk type. Let $T: S \rightarrow S$ be nonexpansive and $\mathscr{F}$ a family of nonexpansive with $T$ commuting maps $S \rightarrow S$ such that for every $f \in \mathscr{F}$ there is a $g \in \mathscr{F}$ with $f \circ g=T$.

THE LAST THEOREM. F has a common fixpoint.

Proof. Consider

$$
\begin{aligned}
Z^{*} & =\{Y \subset S \mid T(Y) \subset Y\}, \\
Z & =\left\{Y \in Z^{*} \mid f(Y) \subset Y \forall f \in \mathscr{F}\right\}
\end{aligned}
$$

and

$$
X=\{M \subset S \mid \varnothing \neq M=\overline{\mathrm{co}} M, T(M) \subset M, f(M) \subset M \forall f \in \mathscr{F}\} .
$$

The map $\varphi(Y) \doteqdot T(Y)$ is decreasing monotone on $Z^{*}$. Since $Z$, is a $\varphi$-subset of $Z^{*}$, It $\varphi$ maps $Z \rightarrow Z$. By Kirk's theorem there is a $T$-fixpoint $x_{M} \in M$ for every $M \in X$. Thus $\left\{x_{M}\right\}$ is a $\varphi$-fixpoint and according to Lemma 2(i) we have $x_{M} \in$ It $\varphi(M)$ and It $\varphi(M)$ must be nonempty. Furthermore we know $\overline{\operatorname{co}} f(\operatorname{It} \varphi(M)) \supset f($ It $\varphi(M))=$ 
It $\varphi(M)$ because of It $\varphi(M)=T(\operatorname{It} \varphi(M))=f \circ g(\operatorname{It} \varphi(M)) \subset f(\operatorname{It} \varphi(M))$. With the aid of Lemma 3 we conclude that $\Psi: M \rightarrow \operatorname{Ceb}_{M} \overline{\operatorname{co}}(\operatorname{It} \varphi(M))$ is a map $X \rightarrow X$. Obviously we have $\operatorname{Ceb}(\operatorname{It} \Psi(S))=\operatorname{It} \Psi(S)$. Hence It $\Psi(S)$ is a singleton consisting of a fixpoint for $\mathscr{F}$.

Corollary (Belluce-Kirk [1]). Let $\mathscr{F}$ be a finite family of commuting nonexpansive maps $S \rightarrow S$. Then $\mathscr{F}$ has a common fixpoint in $S$.

Proof. Use the last theorem for $T=f_{1} \circ f_{2} \circ \cdots \circ f_{n}$, where $\mathscr{F}$ consists of all compositions of different elements of $\left\{f_{1}, \cdots, f_{n}\right\}$.

The last theorem is quite general because whenever a family $\tilde{\mathscr{F}}$ of nonexpansive maps has a common fixpoint $x_{0} \in S$ then we can find a $T$ and a family $\mathscr{F} \supset \tilde{F}$ fulfilling the required conditions. For example define $T(x)=x_{0} \forall x \in S$ and $\mathscr{F}=\mathscr{F} \cup\{T\}$ then obviously $f \circ T=$ $T \forall f \in \tilde{\mathscr{F}}$.

There are very many other theorems which are simple applications of the iteration theorem. One example: the fixpoint theorem of Brodski-Milman [2]. Lim's generalization of the Belluce-Kirk Theorem ([10], [11]) also can be simplified with the iteration theorem. But it seems that Lim's theorem cannot be done without the axiom of choice. All theorems of this paper are theorems of constructive functional analysis although many of them are usually proved with the aid of Zorn's lemma. This seems worthwhile to mention because the interest in constructive analysis has grown with the discovery of Solovay's model [7].

\section{REFERENCES}

1. L. P. Belluce and W. A. Kirk, Fixed-point theorems for families of contraction mappings, Pacific J. Math., 18 (1966), 213-217.

2. M. S. Brodskii and D. P. Milman, Über das Zentrum einer konvexen Menge, Dokl. Akad. Nauk. SSR, 59 (1948), 837-840.

3. Lj. B. Ćirić, A generalization of Banach's contraction principle, Proc. Amer. Math. Soc., 45 (1974), 267-273.

4. N. Dunford and J. T. Schwartz, Linear Operators, New York-London-Sydney. Interscience Publishers, (1958).

5. M. Edelstein, On nonexpansive mappings of uniform spaces, Indag. Math., 27 (1965), 47-51.

6. K. Floret, Eine Bemerkung über a-priori-Fixpunkte nicht-expansiver Abbildungen, Manuscripta Math., 6 (1972), 321-326.

7. H. G. Garnir, Solovay's axiom and functional analysis, Springer Lecture notes, \# 399 (1974), 189-204.

8. W. A. Kirk, A fixed point theorem for mappings which do not increase distances, Amer. Math. Monthly, 72 (1965), 1004-1006. 
9. B. Knaster, Un théorème sur les fonctions d'ensembles, Ann. Soc. Pol. Math., 6 (1927), 133-134.

10. T. C. Lim, A fixed point theorem for families of nonexpansive mappings, Pacific J. Math., 53 (1974), 487-493.

11. - Characterizations of normal structure, Proc. Amer. Math. Soc., 43 (1974), 313-319.

12. J. Reinermann, Fixpunktsätze vom Krasnoselski Typ, Math. Z., 119 (1971), 339-344.

13. B. N. Sadovski, Limit-compact and condensing operators, Russian Math. Survey, 27 (1972), 85-155.

14. A. Tarski, A lattice theoretical fixpoint theorem and its applications, Pacific J. Math., 5 (1955), 285-310.

15. E. Zermelo, Neuer Beweis für die Möglichkeit einer Wahlordnung, Math. Ann., 65 (1908), $107-128$.

Received November 16, 1976

GESAMTHOCHSCHULE

D479 PADERBORN

Germany 



\section{Pacific Journal of Mathematics}

Vol. 68, No. 1

March, 1977

Richard Julian Bagby, On $L^{p}, L^{q}$ multipliers of Fourier transforms . .......

Robert Beauwens and Jean-Jacques Van Binnebeek, Convergence theorems in

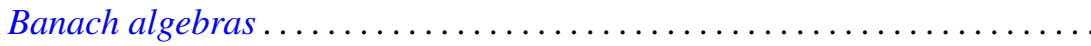

James Cyril Becker, Skew linear vector fields on spheres in the stable

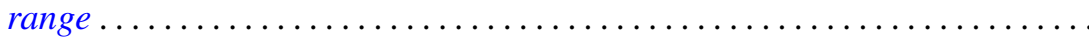

Michael James Beeson, Continuity and comprehension in intuitionistic formal

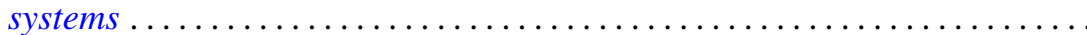

James K. Deveney, Generalized primitive elements for transcendental field

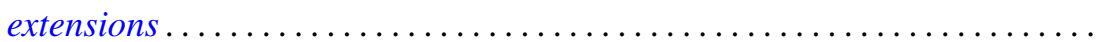

Samuel S. Feder, Samuel Carlos Gitler and K. Y. Lam, Composition properties

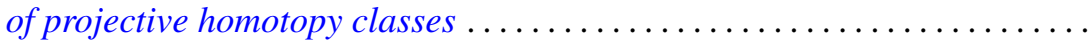

Nathan Jacob Fine, Tensor products of function rings under composition ......

Benno Fuchssteiner, Iterations and fixpoints . . . . . . . . . . . . . .

Wolfgang H. Heil, On punctured balls in manifolds

Shigeru Itoh, A random fixed point theorem for a multivalued contraction

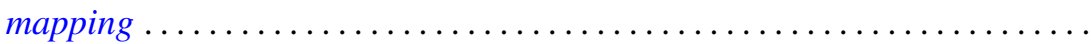

Nicolas P. Jewell, Continuity of module and higher derivations . . . . . . ......

Roger Dale Konyndyk, Residually central wreath products . . . . . . . . . . .

Linda M. Lesniak and John A. Roberts, On Ramsey theory and graphical

parameters.

Vo Thanh Liem, Some cellular subsets of the spheres.

Dieter Lutz, A perturbation theorem for spectral operators

P. H. Maserick, Moments of measures on convex bodies ... . . .

Stephen Joseph McAdam, Unmixed 2-dimensional local domains . .

D. B. McAlister and Norman R. Reilly, E-unitary covers for inverse semigroups...

William H. Meeks, III and Julie Patrusky, Representing codimension-one

homology classes by embedded submanifolds . . .

Premalata Mohapatro, Generalised quasi-Nörlund summability . .

Takahiko Nakazi, Superalgebras of weak-*Dirichlet algebras .

Catherine Louise Olsen, Norms of compact perturbations of operators .

William Henry Ruckle, Absolutely divergent series and isomorphism of

subspaces. II.

Bernard Russo, On the Hausdorff-Young theorem for integral operators .

Arthur Argyle Sagle and J. R. Schumi, Anti-commutative algebras and

homogeneous spaces with multiplications ............

Robert Evert Stong, Stiefel-Whitney classes of manifolds .

D. Suryanarayana, On a theorem of Apostol concerning Möbius functions of

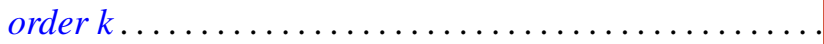

Yoshio Tanaka, On closedness of $C$ - and $C^{*}$-embeddings . . 